\title{
Asymmetry-driven structure formation in pair plasmas
}

\author{
S. M. Mahajan* \\ Institute for Fusion Studies, The University of Texas at Austin, Austin, Texas 78712, USA \\ N. L. Shatashvili ${ }^{\dagger}$ and V. I. Berezhiani \\ Andronikashvili Institute of Physics, Tbilisi 0177, Georgia and Faculty of Exact and Natural Sciences, Department of Physics, \\ Javakhishvili Tbilisi State University, Tbilisi 0128, Georgia \\ (Received 24 August 2009; published 11 December 2009)
}

\begin{abstract}
The nonlinear propagation of electromagnetic waves in pair plasmas, in which the electrostatic potential plays a very important but subdominant role of a "binding glue" is investigated. Several mechanisms for structure formation are investigated, in particular, the "asymmetry" in the initial temperatures of the constituent species. It is shown that the temperature asymmetry leads to a (localizing) nonlinearity that is qualitatively different from the ones originating in ambient mass or density difference. The temperature-asymmetry-driven focusing-defocusing nonlinearity supports stable localized wave structures in 1-3 dimensions, which, for certain parameters, may have flat-top shapes.
\end{abstract}

DOI: 10.1103/PhysRevE.80.066404

PACS number(s): 52.27.Cm, 52.27.Ep, 52.30.Ex, 52.35.Hr

\section{INTRODUCTION}

The pair plasmas consisting of only positive- and negative-charged particles of equal mass have attracted special attention mainly because of the astrophysical applications. In the early universe during the lepton era, ultrarelativistic electron-positron $(e-p)$ pairs contribute largely to the matter contents of the universe [1]. The gamma-ray burststhe most concentrated electromagnetic (em) explosions in the universe-are believed to be related with the enormous energy release in compact regions on short time scales. This energy release leads to the formation of a highly dense optically thick $e-p$ plasma that expands and cools down remaining relativistic [2]. Such pair plasmas exist also in active galactic nuclei, in the relativistic jets [3], and in the pulsar magnetospheres [4].

Although there are many laboratory setups in which $e-p$ pair plasmas are produced [5-8], one of the most interesting recent laboratory accomplishments is the successful creation of "sufficiently" dense pair-ion (pi) plasmas - the first such plasma consisted of equal-mass positive and negative fullerene ions $\left(\mathrm{C}_{60}^{+}\right.$and $\left.\mathrm{C}_{60}^{-}\right)$[9].

Unlike the $e-p$ plasma systems (both of the astrophysical and laboratory variety), the fullerene plasma has a long enough life time that the collective behavior peculiar to the plasma state can be experimentally investigated under controlled conditions. The frequencies associated with the collective modes (plasma frequency, acoustic, and Alfvén frequencies) in such plasmas tend to be rather low. Fortunately, the group of Hatekayama and Oohara have already made considerable progress in the production of the hydrogen, $\mathrm{H}^{+}-\mathrm{H}^{-}$plasmas $[10,11]$. Since the initial report, both the quality and quantity of this light pair-ion plasma has been steadily improving [12].

Since many properties of pair plasmas (a symmetric pair plasma, for instance, cannot sustain charge separation) are

\footnotetext{
*mahajan@mail.utexas.edu

†shatash@ictp.it
}

different from the ordinary electron-ion $(e-i)$ plasma controlled experiments would not only advance fundamental physics but also create a laboratory to simulate and understand a variety of phenomena taking place in astrophysical environments. A basic requirement for long time scale experiments will be that the pair annihilation time scale is many orders of magnitude larger than the plasma period.

Theoretical investigation of pair plasmas have followed two distinct tracks:

(1) The first track emphasizes the special properties that stem from the symmetric (pair particles have the same inertia, temperature, etc.) nature of the pair plasma. This is a highly studied field [13-16] both in the astrophysical as well as the laboratory contexts. In the $e-p$ plasmas, problems such as solitary structure formation are studied for instance in [17], while for the ion pair plasmas much of the linear as well as nonlinear work has been devoted to understanding and interpreting experimental results (see, e.g., $[18,19]$ ), in particular the dispersion curves, described in [9].

(2) The second group of papers deals with a whole lot of interesting phenomena that arise because the symmetry of the pair plasma is mildly broken through some mechanism which creates some disparity between the constituent fluids [20-27]. One of the more interesting results of symmetry breaking is the creation of localized nonlinear structures; the particular properties of the structure will, naturally, depend upon the mode of symmetry breaking.

Although it is natural to imagine that pair plasmas have to be symmetric: that the charges, densities, temperatures, and masses of positively and negatively charged particles are equal. But both observations and experiments indicate that asymmetry may appear, quite naturally, at some stage of their evolution. In fact asymmetry could also be engineered in experiments if such a state could show interesting properties. Different species, not produced in identical conditions [12], for instance, could have different thermal speeds (temperatures). One could also arrange experiments with different setups for different species when, for instance, there are fractions of heavier or lighter ions or there is a mixture of different mass or temperature species with opposite charges. 
This way one could mimic the conditions pertinent to astrophysical pair plasmas.

A much investigated example of broken symmetry is a pair plasma contaminated by a small fraction of charged particles with different mass (lighter or heavier than main species). Symmetry breaking could also occur when the constituent elements of the two fluids have slightly different masses or the fluids have slightly different temperatures. As expected, symmetry breaking induces properties that are different from what pertains for pure pair plasmas. It was shown in [24-26] that pair plasma, contaminated by the heavier immobile ions, can support three-dimensional (3D) stable completely localized structures of em radiation"light-bullets," nondiffracting and nondisspersive em pulses of pancake shape with large density bunching. Existence of such localized structures is not possible in pure pair plasmas. Localized nonlinear structures of em radiation were also found in a hot $e-p$ relativistic plasma containing a small fraction of cold electron-ion component [27]. Similar behavior could be expected in doped (or dust-contaminated) fullerene plasmas in laboratory [28].

The present paper concentrates on establishing the existence of em solitonic structures in pair plasmas that could have been created due to asymmetries of different origin. We will work out the consequences of two classes of symmetry breaking; asymmetry arising from a small temperature difference of the constituent species and the asymmetry arising from a small difference in the species masses. The plasma is assumed to be underdense while the em pulse is longer then characteristic skin length of the plasma. In electron-ion underdense plasma the formation of solitonic structures takes place only at the frequency close to the plasma frequency since in such plasma Raman instabilities dominate the process of soliton formation. In contrast, in pure symmetric pair plasma, ponderomotive forces are same for different species and the excitation of longitudinal waves by the em pulse and Raman instabilities cannot develop. In slightly asymmetric pair plasmas the ponderomotive forces acting on positively and negatively charged species are slightly different; the generation of weak ambipolar electrostatic potential takes place and the effects related to Raman instabilities can be ignored to leading order. This potential plays a fundamental role in structure formation; it acts as the binding "glue" that concentrates matter and radiation in a small region. The nonlinearity due to temperature asymmetry is found to have a focusingdefocusing form (different from the nonlinearities originating in other modes of symmetry breaking) imparting a rich structure to the corresponding solitons.

\section{MODEL}

Let us assume that the velocity distribution of particles is locally a relativistic Maxwellian. Then the dynamics of the fluid of species $\alpha$ ( $\alpha$ represents negative and positive particles of any origin) is contained in the equations (see for details [29]):

$$
\frac{\partial}{\partial t}\left(G_{\alpha} \mathbf{p}_{\alpha}\right)+m_{0 \alpha} c^{2} \nabla\left(G_{\alpha} \gamma_{\alpha}\right)=e_{\alpha} \mathbf{E}+\left(\mathbf{u}_{\alpha} \times \mathbf{\Omega}_{\alpha}\right),
$$

whose curl converts the equation of motion to the vortexdynamical form:

$$
\frac{\partial \boldsymbol{\Omega}_{\alpha}}{\partial t}=\nabla \times\left(\mathbf{u}_{\alpha} \times \boldsymbol{\Omega}_{\alpha}\right),
$$

where $\mathbf{p}_{\alpha}=\gamma_{\alpha} m_{\alpha} \mathbf{u}_{\alpha}$ is the hydrodynamic momentum, $\mathbf{E}$ and $\mathbf{B}$ are the electric and magnetic fields, and $\boldsymbol{\Omega}_{\alpha}=\left(e_{\alpha} / c\right) \mathbf{B}+\nabla$ $\times G_{\alpha} \mathbf{p}_{\alpha}$ is the so-called generalized vorticity. Here $\mathbf{u}_{\alpha}$ denotes he hydrodynamic velocity, $\gamma_{\alpha}=\left(1-\mathbf{u}_{\alpha}^{2} / c^{2}\right)^{-1 / 2}=(1$ $\left.+\mathbf{p}_{\alpha}^{2} / m_{0 \alpha}^{2} c^{2}\right)^{1 / 2}$ is the relativistic factor and $m_{\alpha} G_{\alpha}\left(z_{\alpha}\right)$ $=m_{\alpha} K_{3}\left(z_{\alpha}\right) / K_{2}\left(z_{\alpha}\right)$ is the thermally enhanced "effective mass," $\left[z_{\alpha}=m_{\alpha} c^{2} / T_{\alpha}\right]$, where $K_{\nu}$ are the modified Bessel functions and $m_{\alpha}$ and $T_{\alpha}$ are the particle rest mass and temperature of species $\alpha$, respectively. For nonrelativistic temperatures $\left(T_{\alpha} \ll m_{\alpha} c^{2}\right) \quad G_{\alpha}=1+5 T \alpha / 2 m_{\alpha} c^{2}$ and for the ultrarelativistic temperatures $\left(T_{\alpha} \gg m_{\alpha} c^{2}\right) G_{\alpha}=4 T_{\alpha} / m_{\alpha} c^{2} \gg 1$. Note that the relativistic thermal pressure $P_{\alpha}\left[=\left(n_{\alpha} / \gamma_{\alpha}\right) T_{\alpha}\right.$ in the rest frame with $n_{\alpha}$ being the density in the laboratory frame of the pair fluid] appears through the temperaturedependent factor $G_{\alpha}$ defined by $\gamma_{\alpha} \nabla P_{\alpha}=m_{\alpha} c^{2} n_{\alpha} \nabla G_{\alpha}$. The system of Eqs. (1) and (2) is augmented by the equation of state:

$$
\frac{n_{\alpha} z_{\alpha}}{\gamma_{\alpha} K_{2}\left(z_{\alpha}\right)} \exp \left[-z_{\alpha} K_{2}\left(z_{\alpha}\right)\right]=\text { const }_{\alpha},
$$

which yields the usual results $\left(n_{\alpha}^{\prime} T_{\alpha}^{3 / 2}=\right.$ const in nonrelativistic limit for monoatomic gas and $n_{\alpha}^{\prime} T_{\alpha}^{3}=$ const in ultrarelativistic case for photons).

From Eq. (2) it follows that if the generalized vorticity is initially zero $\left(\boldsymbol{\Omega}_{\alpha}=0\right)$ everywhere in space, it will remain zero for all subsequent times. We assume that before the em radiation is "switched on" the generalized vorticity of the system is zero.

And for both species we have the continuity equation:

$$
\frac{\partial n_{\alpha}}{\partial t}+\nabla \cdot\left(n_{\alpha} \mathbf{u}_{\alpha}\right)=0
$$

To study the nonlinear propagation of intense em wave in a pair plasma (could be relativistically hot) consisting of negative and positive ions, we must couple the equations of motion with Maxwell equations. In terms of the vector (A) and electrostatic $(\phi)$ potentials defined by

$$
\mathbf{E}=-\frac{1}{c} \frac{\partial \mathbf{A}}{\partial t}-\nabla \varphi, \quad \mathbf{B}=\nabla \times \mathbf{A},
$$

the basic equations take the form (Coulomb gauge $\nabla \cdot \mathbf{A}=0$ ):

$$
\frac{\partial^{2} \mathbf{A}}{\partial t^{2}}-c^{2} \Delta \mathbf{A}+c \frac{\partial}{\partial t}(\nabla \varphi)-4 \pi c \mathbf{J}=0
$$

and

$$
\Delta \varphi=-4 \pi \rho,
$$

where for the charge and current densities we have, respectively,

$$
\rho=\sum_{\alpha} e_{\alpha} n_{\alpha}, \quad \mathbf{J}=\sum_{\alpha} e_{\alpha} n_{\alpha} \mathbf{u}_{\alpha} .
$$


Our purpose is to explore the possibility of finding localized structures in such plasmas; the equilibrium state is characterized by charge neutrality: $n_{0}^{+}=n_{0}^{-}$, where $n_{0}^{+}$and $n_{0}^{-}$are the positive and negative charge unperturbed densities. The subscript $\alpha$ hereafter will indicate the negative $(\alpha=-)$ and the positive $(\alpha=+)$ ions. In terms of dimensionless variables:

$$
\begin{gathered}
\mathbf{p}^{ \pm}=\frac{\mathbf{p}^{ \pm}}{m^{-} c}, \quad n^{ \pm}=\frac{n^{ \pm}}{n_{0}^{ \pm}}, \quad T^{ \pm}=\frac{T^{ \pm}}{m^{-} c^{2}}, \quad \mathbf{A}=\frac{|e| \mathbf{A}}{m^{-} c^{2}}, \\
\phi=\frac{|e| \varphi}{m^{-} c^{2}}, \quad \mathbf{r}=\frac{\omega_{-}}{c} \mathbf{r}, \quad t=\omega_{-} t,
\end{gathered}
$$

where $\omega_{-}=\left(4 \pi e^{2} n_{0}^{-} / m^{-}\right)^{1 / 2}$ is the Langmuir frequency of negative species, the entire set of defining equations reads as

$$
\begin{gathered}
\frac{\partial^{2} \mathbf{A}}{\partial t^{2}}-\Delta \mathbf{A}+\frac{\partial}{\partial t}(\nabla \phi)+\left[\frac{n^{-} \boldsymbol{\Pi}^{-}}{\Gamma^{-}}-\frac{n^{+} \boldsymbol{\Pi}^{+}}{\Gamma^{+}}\right]=0, \\
\Delta \phi=n^{-}-n^{+}, \\
\frac{\partial}{\partial t} \Pi^{ \pm}+\nabla \Gamma^{ \pm}=\mp \frac{\partial \mathbf{A}}{\partial t} \mp \nabla \phi, \\
\frac{\partial n^{ \pm}}{\partial t}+\nabla \cdot\left(n^{ \pm} \frac{\boldsymbol{\Pi}^{ \pm}}{\Gamma^{ \pm}}\right)=0,
\end{gathered}
$$

where it was convenient to introduce temperature-dependent momentum $\boldsymbol{\Pi}^{ \pm}=G^{ \pm} \mathbf{p}^{ \pm}$and relativistic factor $\Gamma^{ \pm}=G^{ \pm} \gamma^{ \pm}$ $=\sqrt{\left(G^{ \pm}\right)^{2}+\left(\boldsymbol{\Pi}^{ \pm}\right)^{2}}$. The equations of state for species then read as

$$
\frac{n^{ \pm}}{\Gamma^{ \pm} f\left(T^{ \pm}\right)}=\frac{1}{G_{\infty}^{ \pm} f\left(T_{\infty}^{ \pm}\right)},
$$

with $f\left(T^{ \pm}\right)=\left[T^{ \pm} K_{2}\left(1 / T^{ \pm}\right) / G^{ \pm}\right] \exp \left[G^{ \pm} / T^{ \pm}\right]$and $T_{\infty}^{ \pm}$as the equilibrium temperature of species.

We now discuss the propagation (along the $z$ axis) of a circularly polarized em wave with a mean frequency $\omega_{o}$ and a mean wave number $k_{o}$

$$
\mathbf{A}_{\perp}=\frac{1}{2}(\mathbf{x}+i \mathbf{y}) A\left(\mathbf{r}_{\perp}, z, t\right) \exp \left(i k_{o} z-i \omega_{o} t\right)+\text { c.c. },
$$

where $A(z, t)$ is a slowly varying function of $z$ and $t$ and $\mathbf{x}$ and $\mathbf{y}$ are the standard unit vectors. The choice of circular polarization is dictated by considerations of simplicity (prevents harmonic generation). We shall now follow standard methods to analyze the system. We assume that the longitudinal extent of the pulse is much shorter than its transverse dimensions $\left(\partial A / \partial z \gg \nabla_{\perp} A\right)$. The gauge condition gives us $A_{z}=\left(i / k_{0}\right)\left(\nabla_{\perp} \cdot \mathbf{A}_{\perp}\right) ;\left|A_{z}\right| \ll\left|A_{\perp}\right|$. Consequently the effects related to $A_{z}$ will turn out to be negligibly small. Then, in the slowly varying amplitude approximation, the transverse component of Eq. (12) is integrated yielding

$$
\mathbf{\Pi}_{\perp}^{ \pm}=\mp \mathbf{A}_{\perp},
$$

where the constant of integration is set equal to zero since particle hydrodynamic moments are assumed to be zero at infinity where the field vanishes. Note that for the longitudi- nal motion the equations of motion can be treated one dimensionally.

Longitudinal dynamics is described by the $z$ component of the equation of motion [Eq. (12)] and continuity equation. We note that due to the circular polarization of em wave $\gamma^{ \pm}=\left[1+|A|^{2} /\left(G^{ \pm}\right)^{2}+\left(\Pi_{z}^{ \pm}\right)^{2} /\left(G^{ \pm}\right)^{2}\right]^{1 / 2}$ does not depend on the fast time $\omega_{0}^{-1}$ and all the quantities in Eq. (12) vary on a slow time scale. Therefore, we can introduce the following variables for convenience: $\xi=z-v_{g} t$ and $\tau=t$, where $v_{g}$ $=k_{0} / \omega_{0}$ is the group velocity of the em wave packet. Assuming $v_{g} \partial / \partial \xi \gg \partial / \partial \tau$, straightforward algebra gives the following integral of motion:

$$
G^{ \pm}\left[1+\frac{|A|^{2}}{\left(G^{ \pm}\right)^{2}}+\frac{\left(\Pi_{z}^{ \pm}\right)^{2}}{\left(G^{ \pm}\right)^{2}}\right]^{1 / 2}-v_{g} \Pi_{z} \pm \phi=\text { const }^{ \pm} .
$$

The constants of integration are determined from the boundary conditions: the em fields and plasma momenta vanish at infinity; they are found to be $G_{\infty}^{ \pm}\left(T_{\infty}^{ \pm}\right)$.

In this paper we will deal with transparent plasmas, i.e., $\omega_{0} \gg 1$ and $v_{g} \simeq 1$; such plasmas are found both in astrophysical and laboratory conditions (nontransparent plasma case will be discussed in a future publication). From the continuity equation we have

$$
n^{ \pm}=\frac{\gamma^{ \pm}}{\gamma^{ \pm}-p_{z}},
$$

and straightforward algebra leads to

$$
\begin{gathered}
\gamma^{ \pm}-p_{z}=\frac{G_{\infty}^{ \pm}}{G^{ \pm}}\left[1 \mp \frac{\phi}{G_{\infty}^{ \pm}}\right]^{-1}, \\
\frac{n^{ \pm}}{\gamma^{ \pm}}=\frac{G^{ \pm}}{G_{\infty}^{ \pm}}\left[1 \mp \frac{\phi}{G_{\infty}^{ \pm}}\right]^{-1},
\end{gathered}
$$

where $G_{\infty}^{ \pm} \equiv G_{\infty}^{ \pm}\left(T_{\infty}^{ \pm}\right)$. Equation (17) yields

$$
p_{z}^{ \pm}=\frac{G^{ \pm}}{2 G_{\infty}^{ \pm}}\left[1+\frac{|\mathbf{A}|^{2}}{\left(G^{ \pm}\right)^{2}}-\frac{G_{\infty}^{ \pm}}{\left(G^{ \pm}\right)^{2}}\left(1 \mp \frac{\phi}{G_{\infty}^{ \pm}}\right)^{2}\right]\left[1 \mp \frac{\phi}{G_{\infty}^{ \pm}}\right]^{-1}
$$

and

$$
\gamma^{ \pm}=\frac{G^{ \pm}}{2 G_{\infty}^{ \pm}}\left[1+\frac{|\mathbf{A}|^{2}}{\left(G^{ \pm}\right)^{2}}+\frac{G_{\infty}^{ \pm}}{\left(G^{ \pm}\right)^{2}}\left(1 \mp \frac{\phi}{G_{\infty}^{ \pm}}\right)^{2}\right]\left[1 \mp \frac{\phi}{G_{\infty}^{ \pm}}\right]^{-1},
$$

which allow us to write densities fully in terms of potentials $\phi$ and $\mathbf{A}$ :

$$
n^{ \pm}=\frac{G^{ \pm}}{2 G_{\infty}^{ \pm}}\left[\left(1+\frac{|\mathbf{A}|^{2}}{\left(G^{ \pm}\right)^{2}}\right)\left(1 \mp \frac{\phi}{G_{\infty}^{ \pm}}\right)^{-2}+1\right] .
$$

In pure pair plasma with equal temperature species, the radiation pressure gives equal longitudinal momenta to both the negative and positive ions (since their effective masses are equal $\left[G^{-}=G^{+}=G\right]$ ) and thus does not produce any charge separation $\left(n_{-}=n_{+}\right.$and $\left.\phi=0\right)$ (Berezhiani and Mahajan, 1994). Berezhiani and Mahajan (1995) showed that 
the situation changes by introducing a small fraction of heavy ions; with the symmetry breaking between hot electrons and positrons, it becomes possible to generate a finite $\phi$. Several studies showed the existence of electrostatic potential due to the small fraction of different temperature electrons [20,22]; pair plasmas respond similarly [27].

In [26] it was demonstrated that in the pair-ion plasmas, for which the symmetry is broken by a slight contamination (doping) through a heavier immobile ion, the electrostatic potential is no longer zero and such plasmas can support stable localized em wave structures even in the nonrelativistic limit appropriate to the current and near future laboratory experiments. As we will see below, the creation of electrostatic potential is also possible due to the difference in initial temperatures for different (major constituent) species (yielding the difference in effective masses of species).

\section{FORMATION OF LOCALIZED STRUCTURES IN PAIR PLASMAS WITH TEMPERATURE ASYMMETRY}

We introduce the "asymmetry" through temperature difference between the two species. The existence of temperature asymmetry is both experimentally and observationally justified (see the references given in the introduction) and leads to different effective masses even though the real masses are equal $\left(m^{-}=m^{+}\right)$. We would expect that this asymmetry in effective masses would lead to effects similar to the ones studied for ordinary $e-i$ plasmas. As a first step we assume that temperatures are only slightly different

$$
\frac{G_{\infty}^{-}}{G_{\infty}^{+}}=\beta \neq 1,
$$

where $\beta$ is close to 1 so that we can have small but finite $\phi$.

This assumption lets Eqs. (20) to be written as

$$
\begin{gathered}
\frac{n^{+}}{\gamma^{+}}=\frac{G^{+}}{G_{\infty}^{+}}\left[1-\frac{\phi}{G_{\infty}^{+}}\right]^{-1}=\frac{g^{+}}{1-\beta \hat{\phi}}, \\
\frac{n^{-}}{\gamma^{-}}=\frac{G^{-}}{G_{\infty}^{-}}\left[1+\frac{\phi}{G_{\infty}^{-}}\right]^{-1}=\frac{g^{-}}{1+\hat{\phi}},
\end{gathered}
$$

where the following normalization $g^{ \pm}=\left(G^{ \pm} / G_{\infty}^{ \pm}\right)$and $\hat{\phi}$ $=\phi / G_{\infty}^{-}$were used. Equation (23), written fully in terms of potentials $\phi, A$, relativistic factors $G^{ \pm}$, and $\beta$, transforms to

$$
\begin{gathered}
n^{+}=\frac{1}{2}\left[1+\frac{\left(g^{+}\right)^{2}+\beta^{2}|\hat{A}|^{2}}{(1-\beta \hat{\phi})^{2}}\right], \\
n^{-}=\frac{1}{2}\left[1+\frac{\left(g^{-}\right)^{2}+|\hat{A}|^{2}}{(1+\hat{\phi})^{2}}\right],
\end{gathered}
$$

where $\hat{A}=A / G_{\infty}^{-}$. From now on we will omit the $\hat{\cdots}$ and it will be assumed that $\phi$ and $A$ are normalized to $G_{\infty}^{-}$.

For clarity of exposition we will not attempt to analyze the general case confining ourselves to the two extreme limits: nonrelativistic and super-relativistic temperatures.

To close Maxwell equations, we need to evaluate $\mathbf{J}^{ \pm}$ $=n^{ \pm} \boldsymbol{\Pi}^{ \pm} / \Gamma^{ \pm}$, the current that goes into Eq. (10). Using Eqs.
(25) and (26), and the normalized version of Eq. (16)

$$
g^{ \pm} \mathbf{p}_{\perp}^{ \pm}=\mp \mathbf{A}
$$

the expression for the total current is easily derived to be

$$
\mathbf{J}_{\perp}=-\left[\frac{1}{1-\beta \phi}+\frac{1}{1+\phi}\right] \mathbf{A} .
$$

Using the quasineutrality condition $n^{+}=n^{-}$(characteristic length scale of wave $L \gg 1$ ) and plugging it into the Eqs. (27) and taking into account that for a transparent plasma heating of both fluids is very weak (implying $g^{ \pm} \sim 1$ ), we can derive $\phi \sim(1-\beta) \psi\left(|A|^{2}\right)$ [where $\left.\psi\left(|A|^{2}\right) \leq 1\right]$. Putting all the pieces together [evaluating the current, actually the $\left(\mathbf{J}_{\perp}-2 \mathbf{A}\right)$ in our notation] the nonlinear term in Eq. (10) is expressible as

$$
\mathrm{NL}=\frac{1}{1-\beta \phi}+\frac{1}{1+\phi}-2 \simeq-\phi[(1-\beta)-2 \beta \phi],
$$

where we do not neglect $\phi$ with respect to $(1-\beta) \ll 1$. To complete Maxwell's equations, we have to relate the electrostatic potential $\phi(<1)$ with its source, in this case, the temperature difference between the species.

\section{A. Super-relativistic temperature pair plasmas}

For super-relativistic temperatures $T^{ \pm} \gg 1$ and $G^{ \pm}=4 T^{ \pm}(\gg 1), g^{ \pm}=T^{ \pm} / T_{\infty}^{ \pm}$and Eq. (14) reads as

$$
\frac{n^{ \pm}}{\gamma^{ \pm}}=\left(\frac{T^{ \pm}}{T_{\infty}^{ \pm}}\right)^{3}=g^{ \pm 3},
$$

which, in conjunction with Eqs. (25), (26), and (31), yields

$$
g^{+}=\frac{1}{(1-\beta \phi)^{1 / 2}}, \quad g^{-}=\frac{1}{(1+\phi)^{1 / 2}},
$$

evaluating the effective mass $G^{ \pm}$self-consistently in terms of $\phi$. Straightforward algebra and the use of quasineutrality condition yields

$$
\phi \simeq \frac{|A|^{2}}{3}[(1-\beta)-2 \beta \phi],
$$

leading, finally, to

$$
\phi=\frac{(1-\beta)}{2} \frac{\kappa|A|^{2}}{\left(1+\beta \kappa|A|^{2}\right)} \quad \text { with } \quad \kappa \equiv \frac{2}{3} .
$$

We see that $\phi \sim(1-\beta)$ when $|A|^{2} \gg 1$ and $\phi \leq(1-\beta)$ when $|A|^{2} \leq 1$ and our estimation of nonlinear term [Eq. (30)] remains valid.

Note that with electrostatic potential defined by Eq. (33) one learns that the heating or cooling of both fluids is weak $\left[g^{+} \sim(1+0.5 \beta \phi), g^{-} \sim(1-0.5 \phi)\right]$. Also from Eqs. (27) the species densities come out to be

$$
n^{ \pm} \sim 1+\frac{\beta}{2}|A|^{2}
$$

\section{B. Nonrelativistic temperature pair plasmas}

For nonrelativistic temperatures $T^{ \pm}, T_{\infty}^{ \pm} \ll 1$ and $G^{ \pm}=1$ $+5 T^{ \pm} / 2$. In this limit the relevant relations are 


$$
\frac{n^{ \pm}}{\gamma^{ \pm}}=\left(\frac{T^{ \pm}}{T_{\infty}^{ \pm}}\right)^{3 / 2}, \quad g^{ \pm} \simeq 1+\frac{5}{2}\left(T^{ \pm}-T_{\infty}^{ \pm}\right)
$$

and

$$
\beta=\frac{G_{\infty}^{-}}{G_{\infty}^{+}} \simeq 1+\frac{5}{2}\left(T_{\infty}^{-}-T_{\infty}^{+}\right) .
$$

Straightforward but tedious algebra leads us to

$$
g_{ \pm}=1+H_{ \pm},
$$

where

$$
H_{+}=\frac{5}{2} \beta T_{\infty}^{+} \phi \quad \text { and } \quad H_{-}=-\frac{5}{2} T_{\infty}^{-} \phi .
$$

From Eqs. (37) and (38) we find

$$
\begin{aligned}
& g_{+}+g_{-} \simeq 2+(\beta-1) \phi, \\
& g_{+}-g_{-} \simeq \frac{5}{2} \phi T_{\infty}^{-}(1+\beta),
\end{aligned}
$$

from which

$$
g_{+}^{2}-g_{-}^{2} \simeq \phi T_{\infty}^{-}(1+\beta),
$$

readily follows. Repeating the same procedure as we adopted earlier, we may derive

$$
\phi=\frac{(1-\beta)}{2} \frac{\kappa|A|^{2}}{\left(1+\beta \kappa|A|^{2}\right)}, \quad \text { with } \quad \kappa \equiv \frac{1}{2},
$$

an expression that has the same general form as Eq. (33) that pertains to the super-relativistic temperature regime. In fact, the two limits are very similar-for either case the plasma heating or cooling is weak and the density bunching $n^{ \pm} \sim 1$ $+\beta|A|^{2} / 2$.

\section{LOCALIZED STRUCTURES}

Our interest, here, is to delineate the conditions for the existence of localized structures in pair plasmas. For the temperature-asymmetry-driven system, the perpendicular current is assembled from Eqs. (33) and (41):

$$
\begin{aligned}
J_{\perp} & \simeq-\{2-\phi[(1-\beta)-2 \beta \phi]\} A \\
& =-\left[2-\frac{(1-\beta)^{2}}{2} \frac{\kappa|A|^{2}}{\left(1+\beta \kappa|A|^{2}\right)^{2}}\right] A,
\end{aligned}
$$

with $\kappa=1 / 2$ for nonrelativistic temperatures and $\kappa=2 / 3$ for relativistic temperatures. The final equation for the vector potential [equivalent to the final defining Eq. (43) of [25]], derived from Eq. (10) and the preceding expression of current, reads

$$
\begin{aligned}
& 2 i \omega_{0} \frac{\partial A}{\partial \tau}+\frac{1+\beta}{\omega_{0}^{2}} \frac{\partial^{2} A}{\partial \xi^{2}}+\nabla_{\perp}^{2} A \\
& +\frac{(1-\beta)^{2}}{2} \frac{\kappa|A|^{2}}{\left(1+\beta \kappa|A|^{2}\right)^{2}} A=0, \\
& \text { with } \epsilon^{2} \equiv \frac{1}{4}(1-\beta)^{2} \ll 1,
\end{aligned}
$$

where we have redefined $m_{-}$as $m_{-} \rightarrow m_{-} G_{\infty}^{-}$that introduces the effective mass for negatively charged ions. The wave frequency $\omega_{0}$ satisfies the dispersion relation: $\omega_{0}^{2}=k_{0}^{2}+(1$ $+\beta$ ) [in dimensional units this reads as: $\omega_{0}^{2}=k_{0}^{2} c^{2}+(1+\beta) \omega_{-}^{2}$ ]. In Eq. (43) the weak dependence on the transverse coordinates has been retained. Note that in spite of the fact that $\partial A / \partial \xi \gg \nabla_{\perp} A$ the second and the third terms can be comparable because of the "transparent plasma" $\left(\omega_{0}^{2} \gg 2\right)$ condition [30].

With self-evident renormalization, Eq. (43) can be written as

$$
i \frac{\partial A}{\partial \tau}+\frac{\partial^{2} A}{\partial \xi^{2}}+\nabla_{\perp}^{2} A+F\left(|A|^{2}\right) \cdot A=0,
$$

with the nonlinearity function given by

$$
F\left(|A|^{2}\right)=\frac{|A|^{2}}{\left(1+|A|^{2}\right)^{2}} .
$$

Equation (44) is nothing but the nonlinear Schrödinger equation (NSE) with a saturating nonlinearity. The saturation function $F\left(|A|^{2}\right.$ ) constitutes a new type; it has an unusual form in the sense that in the ultrarelativistic case $|A|^{2} \gg 1$ it tends to vanish. NSE with such a "vanishing saturation potential" has not been derived and reported for any known physical system.

From the refractive index $\delta n_{n l}=F(I)\left(I=|A|^{2}\right.$ is the intensity of the em field), we may deduce that the plasma is selffocusing $\left[d\left(\delta n_{n l}\right) / d I>0\right]$ provided $I<1$ while it becomes defocusing $\left[d\left(\delta n_{n l}\right) / d I<0\right]$ for higher intensities $(I>1)$. For a localized intense em pulse with a peak intensity $I_{m}>1$, the medium, thus, reacts differently to different parts of the em pulse-focusing the peak region while defocusing the wings.

An immediate consequence of the focusing-defocusing saturating nonlinearity (originating from the temperatureasymmetry-driven mechanism) can be illustrated by considering a modulation instability of quasimonochromatic em wave. Equation (44) is satisfied by the plane wave solution $A=A_{0} \exp \left[i \tau F\left(\left|A_{0}\right|^{2}\right)\right]+$ c.c.. The standard stability analysis then shows that a linear modulation with frequency $\Omega$ and wave number $\mathbf{K}$ obeys the dispersion relation $\Omega^{2}=\mathbf{K}^{2}\left[\mathbf{K}^{2}\right.$ $\left.-2 A_{0}^{2}\left(1-A_{0}^{2}\right) /\left(1+A_{0}^{2}\right)^{3}\right]$ which exhibits a purely growing mode if $A_{0}<1$ and $K<K_{c r}=\sqrt{2 A_{0}^{2}\left(1-A_{0}^{2}\right) /\left(1+A_{0}^{2}\right)^{3}}$, while for the ultrarelativistic case $\left(A_{0}>1\right)$, there is no modulation instability. One can expect that the modulation instability of moderately intense field $\left(A_{0}<1\right)$ in the nonlinear stage will lead to the break up of the field into solitonlike pulses with a characteristic length corresponding to the optimum scale of instability $\left(\sim \sqrt{2} / K_{c r}\right)$.

In what follows we investigate the possibility of finding stable solitonic solutions of Eq. (44) under a variety of conditions. For stationary solitons, we look for solutions that are "spherical" symmetric: $A=A(r) \exp (i \lambda)$, where $\lambda$ is a constant measuring the nonlinear frequency shift. Notice that the comoving coordinate $(\xi)$ can be treated on an equal footing with the spatial coordinate $\left(\mathbf{r}_{\perp}\right)$. In terms of the radial variable $r=\left(\mathbf{r}_{\perp}^{2}+\xi^{2}\right)^{1 / 2}$, Eq. (44) reduces to an ordinary differential equation that cannot be analytically solved. However, to better understand the results of possible simulations it is 


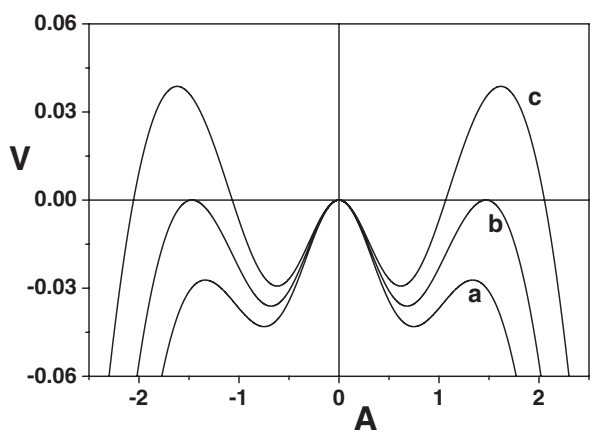

FIG. 1. The effective potential $V$ vs the amplitude $A$ for different values of the nonlinear frequency shift $\lambda$. The curve "a" corresponds to $\lambda>\lambda_{c r}^{(1 \mathrm{D})} \simeq 0.2162$, the curve " $\mathrm{b}$ " has $\lambda=\lambda_{c r}^{(1 \mathrm{D})}$, and the curve "c" corresponds to $0<\lambda<\lambda_{c r}^{(1 \mathrm{D})}$.

helpful to rewrite it (after trivial manipulations) as the equation describing a "particle" moving with friction in the potential:

$$
\frac{d}{d r}\left[\left(\frac{d A}{d r}\right)^{2}+V(A)\right]=-\frac{2(D-1)}{r^{D-1}}\left(\frac{d A}{d r}\right)^{2},
$$

where the effective potential is $V(A)=-\lambda A^{2}+\ln \left(1+A^{2}\right)$ $-A^{2} /\left(1+A^{2}\right)$. Here $D(=1,2,3)$ dimension of the problem.

The profile of the potential for different values of the nonlinear frequency shift $\lambda$ is presented in Fig. 1: (1) curve "a" in this plot corresponds to $\lambda>\lambda_{c r}^{(1 \mathrm{D})} \simeq 0.2162$, (2) $\lambda$ $=\lambda_{c r}^{(1 D)}$ for the curve " $b$," and (3) the potential in the range $0<\lambda<\lambda_{c r}^{(1 \mathrm{D})}$ is given in curve "c." The solitary solutions correspond to the effective particle which at the moment $r$ $=0$ rests at a point with coordinate $A_{m}$. Then it rolls down $(r>0)$, dissipates "energy" and approaches asymptotically $(r \rightarrow \infty)$ the potential maximum at $A=0$. It is obvious that solitary solution cannot exist for $\lambda>\lambda_{c r}^{(1 D)}$ while for $\lambda$ $=\lambda_{c r}^{(1 \mathrm{D})}$ solitary solution does exist.

However in the range covered in $\mathrm{c}\left(0<\lambda<\lambda_{c r}^{(1 \mathrm{D})}\right)$ solitary wave solution exists in more than one dimension; the corresponding $A_{m}$ for a given eigenvalue $\lambda$ has to be found numerically. Notice that in one dimension, the particle motion becomes conservative significantly simplifying the problem.

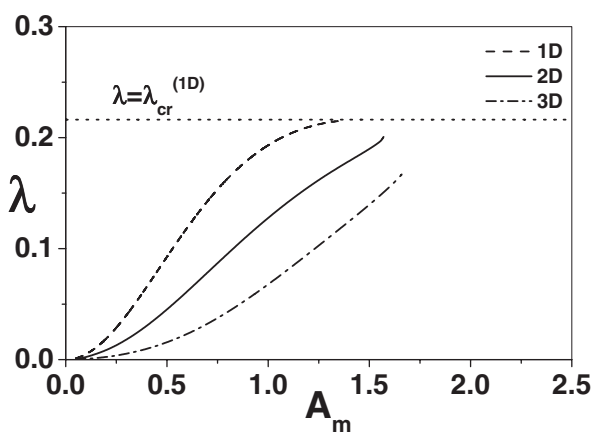

FIG. 2. Nonlinear dispersion relations: the effective eigenvalue $\lambda$ as a function of $A_{m}$. The boundary line (dotted) corresponds to critical value $\lambda=\lambda_{c r}^{(1 \mathrm{D})}$ analytically found only for $1 \mathrm{D}$. The other three lines represent, respectively, the $1 \mathrm{D}, 2 \mathrm{D}$, and $3 \mathrm{D}$ dispersion relations.

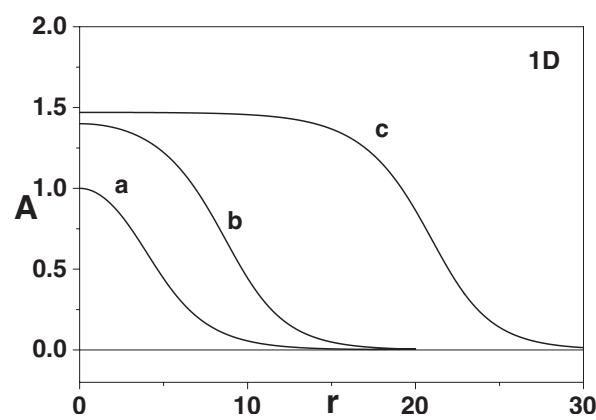

FIG. 3. Stationary soliton solution for $1 \mathrm{D}$ for different critical eigenvalues. Plot a corresponds to $\lambda_{c r}=0.19315$ with $A_{m}=1$; plot b corresponds to $\lambda_{c r}=0.21583$ with $A_{m}=1.4$ and plot c corresponds to $\lambda_{c r}=0.21622$ with $A_{m}=1.47$, respectively. The plot c represents the flat-top soliton solution.

By demanding $V(A)=0$ we find the relationship $\lambda=\ln (1$ $\left.+A_{m}^{2}\right) / A_{m}^{2}-1 /\left(1+A_{m}^{2}\right)$. It can be established analytically that the growing slope of this relation defines the amplitude of the soliton $A_{m}$. Corresponding nonlinear dispersion relation $\lambda=\lambda\left(A_{m}\right)$ is exhibited in Fig. 2 [curve one-dimensional (1D)]. One can see from these figures that the $1 \mathrm{D}$ solution is restricted from above: $A_{m} \leq A_{m c r} \cong 1.4506$. For small amplitudes Eq. (44) reduces to the standard NSE with a cubic nonlinearity, and 1D soliton solution can be found analytically [see [26] for cold plasma case when $n=n(T)$ ].

Profiles for large amplitude 1D solitons are exhibited in Fig. 3. One can see that as $A \rightarrow A_{m c r}\left(\lambda \rightarrow \lambda_{c r}^{(1 \mathrm{D})}\right)$ the profile of the central part of the soliton flattens and widens at the top. The existence of flat-top soliton can be explained by the peculiarities of our focusing-defocusing nonlinearity: the top part of the pulse with $A>1$ lies in the defocusing region with a tendency for diffraction while the wings of the soliton are in the focusing region preventing the total spread of the pulse. It is interesting to remark that for $\lambda>\lambda_{c r}^{(1 \mathrm{D})}$ the system supports existence of the dark soliton which is an antisymmetric function of coordinate with zero intensity at its center. The dark soliton corresponds to the particle starting at the right maximum of the curve "c" and going asymptotically toward the left maximum of the potential. Background intensity of the field $\left|A_{0}\right|$ is bounded from below $\left(\left|A_{0}\right|>A_{m c r}\right)$ but is not restricted from above. Thus, a dark soliton with arbi-

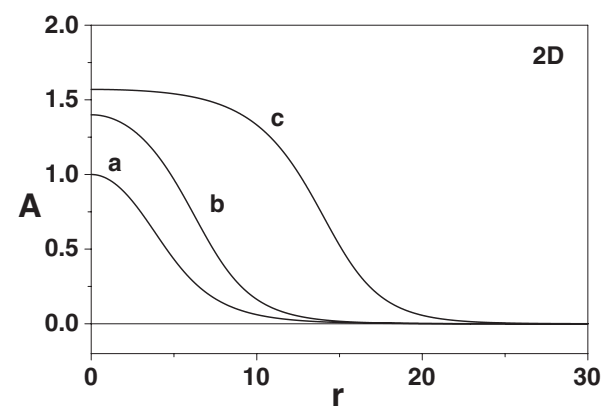

FIG. 4. Stationary soliton solution for 2D for different critical eigenvalues. Plot a corresponds to $\lambda_{c r}=0.12789382$ with $A_{m}=1$; plot b corresponds to $\lambda_{c r}=0.17891793$ with $A_{m}=1.4$ and plot $\mathrm{c}$ corresponds to $\lambda_{c r}=0.20299496$ with $A_{m}=1.57$ respectively. The plot $\mathrm{c}$ represents the flat-top soliton solution. 


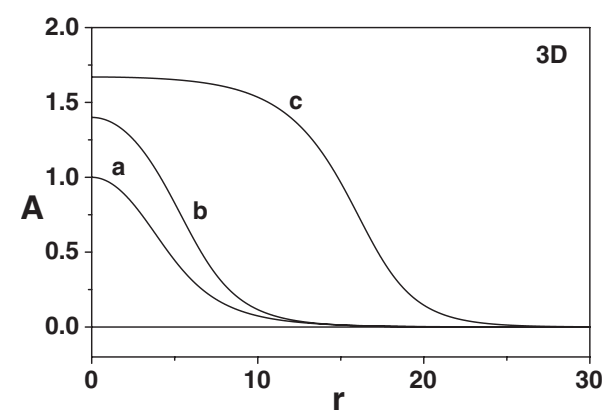

FIG. 5. Stationary soliton solution for 3D for different critical eigenvalues. Plot a corresponds to $\lambda_{c r}=0.6774722$ with $A_{m}=1$; plot b corresponds to $\lambda_{c r}=0.12451945$ with $A_{m}=1.4$ and plot c corresponds to $\lambda_{c r}=0.19222242$ with $A_{m}=1.67$, respectively. The plot $\mathrm{c}$ represents the flat-top soliton solution.

trarily strong background intensity is possible in 1D. At the critical frequency shift $\left(\lambda=\lambda_{c r}^{(1 \mathrm{D})}\right)$ the dark and bright solitons can coexist. The coexistence of these solitons is mainly due to the particular type of nonlinearity encountered in the present model. Detailed discussion of the properties of dark solitons is beyond the intended scope of this paper.

In two-dimensional (2D) and $3 \mathrm{D}$, the nonzero friction force forces the corresponding critical values of $\lambda$ to be less than $\lambda_{c r}^{(1 \mathrm{D})}$ found for the 1D case. In Fig. 2, the relevant curves corresponding to numerically obtained dispersion relations for 2D and 3D are displayed. Fundamental solitary solutions (without zero nodes) are shown in Figs. 4 and 5; in all these examples one fundamental feature of the soliton persists, namely, that near the critical eigenvalues, the profile is endowed with the flat-top shape.

The stability of the obtained solutions can be tested by applying the Vakhitov and Kolokolov criterion (see [31] and references therein) according to which the soliton is stable if $\partial N / \partial \lambda>0$, where $N=\int d \mathbf{r}_{\perp} d \xi|A|^{2}$ is the soliton energy (photon number). We found that in $1 \mathrm{D}$ the photon number is always a growing function of $\lambda$ implying that such solitons are stable against small perturbations.

In Fig. 6 we show the dependence of the photon number on the amplitude $A_{m}$ for $2 \mathrm{D}$ and $3 \mathrm{D}$ solitons. In contrast to the $1 \mathrm{D}$ case, for the soliton to exist in higher dimensions, its energy $N$ must exceed a certain critical value $N_{c r}$. For a 2D case, the photon number must exceed the threshold energy

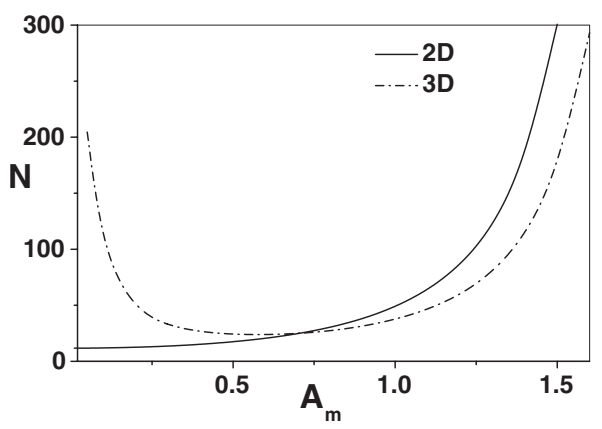

FIG. 6. The dependence of the photon number $N$ on the amplitude $A_{m}$ for 2D and 3D. Normalized photon numbers correspond to $N^{(2 \mathrm{D})}$ (solid line) and $10^{-1} N^{(3 \mathrm{D})}$ (dashed line). For 2D (3D) the threshold energy for the existence of soliton is $N_{c r}=11.6$ (236.8).
$N_{c r}=11.6$ for the em wave to enter the self-guiding solitonic regime. Since $\partial N / \partial A_{m}>0$, then $\partial N / \partial \lambda>0$ because $A_{m}$ is a growing function of $\lambda$ (see Fig. 2). Thus the $2 \mathrm{D}$ soliton with its power above the critical power is always stable. In $3 \mathrm{D}$ $\partial N / \partial A_{m}>0$ provided $N>N_{c r}=236.8$ and $A_{m}>0.6$.

We have demonstrated that the pair plasmas with asymmetry in initial temperatures of its constituents can support stable large amplitude localized em wave structures. These structures, available in arbitrary (1-3) dimensions, have flattop shapes for certain range of parameters. This result is particularly interesting for laboratory conditions. Since even a small difference (very small) in temperature may be as effective in symmetry breaking as the Baryonic correction in early universe, one could readily engineer the laboratory plasmas to mimic the cosmic conditions.

According to Eq. (34) the total plasma density in the pulse localization area increases dramatically for relativistically intense pulses. Notice that the general results of the analysis, as well as Eq. (44) are valid both for ultrarelativistic and nonrelativistic temperatures, and hence warrants applications to both astrophysical and laboratory plasmas.

\section{MASS ASYMMETRY BETWEEN SPECIES}

In this section we explore another obvious source of asymmetry between species-a slight difference in the masses of positive and negative-charged particles. This mechanism is different from the one discussed above; the mass asymmetry is initially given and is fixed as distinct from the dynamical asymmetry created due to temperature differences. Such a plasma can be created by the injection of appropriate ion beams into a trap. Electron-hole plasma in certain semiconductors or $e-p$ collider plasma created by slightly different Lorentz factor beams are also possible examples of such system. Appropriate conditions for such plasma production could readily appear in dusty plasmas, as well as in astrophysical jets, and pulsar magnetospheres.

Much of the framework for investigating this mechanism has already been described. For an unmagnetized plasma, one can use the dimensionless Eqs. (10) and (11) in which the Lorentz factors of negatively and positively charged particles are, respectively, changed to $\gamma^{-}=\left[1+\left(\mathbf{p}^{-}\right)^{2}\right]^{1 / 2}$ and $\gamma^{+}$ $=\delta^{-1}\left[\delta^{2}+\left(\mathbf{p}^{+}\right)^{2}\right]^{1 / 2}$, with $\delta=m^{+} / m^{-}$being the ratio of positive $\left(\mathrm{m}^{+}\right)$and negative $\left(\mathrm{m}^{-}\right)$particle masses.

For a circularly polarized em wave with wave frequency $\omega_{0}^{2}=k_{0}^{2}+\left(1+\delta^{-1}\right)$ one can readily show that $\mathbf{P}_{\perp}^{ \pm}=\mp \mathbf{A}[$ the equivalent of Eq. (16)]. Then the standard treatment for a transparent plasma $\left[\omega_{0} \gg\left(1+\delta^{-1}\right)^{1 / 2}, v_{g}=k_{0} / \omega_{0} \simeq 1\right]$ yields the following relations [equivalent of Eqs. (25)-(27)]:

$$
\frac{n^{-}}{\gamma^{-}}=\frac{1}{1+\phi}, \quad \frac{n^{+}}{\gamma^{+}}=\frac{\delta}{\delta-\phi}
$$

and

$$
n^{-}=\frac{1}{2}\left[1+\frac{1+|A|^{2}}{(1+\phi)^{2}}\right], \quad n^{+}=\frac{1}{2}\left[1+\frac{\delta^{2}+|A|^{2}}{(\delta-\phi)^{2}}\right],
$$

leading to 
$2 i \omega \frac{\partial A}{\partial \tau}+\frac{\left(1+\delta^{-1}\right)}{\omega^{2}} \frac{\partial^{2} A}{\partial \xi^{2}}+\nabla_{\perp}^{2} A+A \phi\left[\frac{1}{1+\phi}-\frac{1}{\delta(\delta-\phi)}\right]=0$.

For small mass differences $\delta=1+\eta(\eta \ll 1)$ and for the characteristic length $L \gg 1$, the potential $\phi$ may be calculated to be

$$
\phi=\frac{\eta|A|^{2}}{2\left(1+|A|^{2}\right)}
$$

explicitly displaying that $\phi$ is proportional to $\eta$, i.e., $\phi \ll 1$ for $\eta \ll 1$ [compare with Eqs. (33) and (41)]. Equation (49) with Eq. (50), with appropriate normalization of the variables and inclusion of transverse field variations, constitute an NLSE (44) with the following saturating nonlinearity function ( $\eta$ will be absorbed in the normalization):

$$
F\left(|A|^{2}\right)=1-\frac{1}{\left(1+|A|^{2}\right)^{2}} .
$$

Notice that this form of saturation nonlinearity function coincides with the one obtained in [24] for $e$ - $p$ plasma with a small fraction of heavy ions. The reader may consult Ref. [25] for detailed analysis of the system; here we will just summarize the salient features. Equation (44) [with Eq. (51)] admits a spherically symmetric solitary wave solution, i.e., the "light bullet," a concentration of mass and energy. And if the bullet $A_{m}>0.7$, this bullet is stable. These light bullets are found to be exceptionally robust [32]: they can emerge from a large variety of initial field distributions and are remarkably stable. The total plasma density variation associated with the soliton $\delta n \sim A^{2}$ is large for $A^{2} \gg 1$; the solitons with ultrarelativistic amplitudes create a large concentration of density.

Thus, the system of pair plasma with slight initial mass asymmetry between species supports the existence of long lived objects-light bullets which carry large amounts of mass and energy exactly the same way as the pair plasmas with small fraction of heavier ions [24-26].

The saturating nonlinearity [Eq. (45)] caused by an initial temperature asymmetry seems to be of a new type (vanishing for intense pulses); it differs quite fundamentally from the one found in [24] (identical to the one originating in a initial mass asymmetry). One would think that the temperature asymmetry, manifesting finally as effective mass asymmetry, would be qualitatively similar to the initial mass asymmetry. But it is not so. Fortunately we could trace the cause of the difference.
The first step in the chain does appear to translate the temperature asymmetry [see Eq. (24)] into a difference in the effective masses $G^{ \pm} m^{ \pm}$. But in reality this mass asymmetry is dynamical and dependent on the scalar potential $\phi$, $m_{\mathrm{eff}}^{+} / m_{\mathrm{eff}}^{-} \sim(1-\epsilon+0.5 \nu \phi)$ while the one originating in an initial mass asymmetry $(\delta=1+\eta)$ is constant in space time and never leads to heating or cooling. It is of utmost significance to realize that the electrostatic potential $\phi$ is important not only for maintaining the nonlinearity but also to create dynamical temperature asymmetry for hot plasma conditions; it is the latter consequence that may lead to qualitatively new and interesting phenomena in such a state of matter.

As a general conclusion we must state that the results of Sec. V are valid only for cold plasmas. For initially hot plasmas one must take into account the temperature inhomogeneity effects (discussed earlier in the paper) that change the nature of the nonlinearity and hence the localization characteristics of the em waves. However it is worth mentioning that the main property of density bunching and energy localization is always there in pair plasmas with different type initial asymmetries; it is just the character of localization that changes with origin of the asymmetry.

\section{SUMMARY AND CONCLUSIONS}

The main result of this paper is that a fundamentally new type of saturating focusing-defocusing nonlinearity is derived for a physical system. This composite nonlinearity originating in a small temperature asymmetry in the constituent fluids of a pair plasma promises the existence of interesting structures that intense electromagnetic waves can acquire in such plasmas. We have discussed an illustrative example where different parts of a high amplitude pulse are effected differently - the simultaneous expansion of the peak region and scrunching of the wings imparts a flat top shape to the pulse. Most consequences of this type of nonlinearity are yet to be worked out.

\section{ACKNOWLEDGMENTS}

We acknowledge our special debt to the Abdus Salam International Centre for Theoretical Physics, Trieste, Italy. The work of S.M.M. was supported by (U.S.) DOE Contract No. DE-FG 03-96ER-54366. The work of N.L.S. and V.I.B. was partially supported by ISTC Project No. G-1366 and Georgian NSF grant projects GNSF 69/07 (Grant No. GNSF/ ST06/4-057) and GNSF 195/07 (Grant No. GNSF/ST07/4191).
[1] S. Wineberg, Gravitation and Cosmology: Principles and Applications of the General Theory of Relativity (Wiley and Sons, New York, 1972).

[2] M. J. Rees and P. Mészáros, Mon. Not. R. Astron. Soc. 258, 41 (1992); P. Mészáros and M. J. Rees, Astrophys. J. 405, 278 (1993); 418, L5 (1993); R. Sari and T. Piran, ibid. 485, 270
(1997).

[3] M. C. Begelman, R. D. Blandford, and M. J. Rees, Rev. Mod. Phys. 56, 255 (1984).

[4] P. A. Sturrock, Astrophys. J. 164, 529 (1971); M. A. Ruderman and P. G. Sutherland, ibid. 196, 51 (1995); F. C. Michel, Theory of Neutron Star Magnetospheres (University of Chi- 
cago Press, Chicago, 1991).

[5] C. M. Surko, M. Leventhal, and A. Passner, Phys. Rev. Lett. 62, 901 (1989); C. M. Surko and T. J. Murphy, Phys. Fluids B 2, 1372 (1990); R. G. Greaves and C. M. Surko, Phys. Plasmas 4, 1528 (1997).

[6] E. P. Liang, S. C. Wilks, and M. Tabak, Phys. Rev. Lett. 81, 4887 (1998); B. Shen and J. Meyer-ter-Vehn, Phys. Rev. E 65, 016405 (2001).

[7] J. W. Shearer et al., Phys. Rev. A 8, 1582 (1973); V. I. Berezhiani, D. D. Tskhakaya, and P. K. Shukla, ibid. 46, 6608 (1992); V. I. Berezhiani, D. P. Garuchava, and P. K. Shukla, Phys. Lett. A 360, 624 (2007).

[8] T. E. Cowan et al., Laser Part. Beams 17, 773 (1999); C. Gahn et al., Appl. Phys. Lett. 77, 2662 (2000); H. Chen, S. C. Wilks, J. D. Bonlie, E. P. Liang, J. Myatt, D. F. Price, D. D. Meyerhofer, and P. Beiersdorfer, Phys. Rev. Lett. 102, 105001 (2009).

[9] W. Oohara and R. Hatakeyama, Phys. Rev. Lett. 91, 205005 (2003); W. Oohara, D. Date, and R. Hatakeyama, ibid. 95, 175003 (2005).

[10] W. Oohara, Y. Kuwabara, and R. Hatakeyama, Phys. Rev. E 75, 056403 (2007).

[11] W. Oohara and R. Hatakeyama, Phys. Plasmas 14, 055704 (2007).

[12] R. Hatakeyama (private communication).

[13] M. E. Gedalin, J. G. Lominadze, L. Stenflo, and V. N. Tsitovich, Astrophys. Space Sci. 108, 393 (1985); P. K. Shukla, N. N. Rao, M. Y. Yu, and N. L. Tsintsadze, Phys. Rep. 131, 1 (1986).

[14] T. Cattaert, I. Kourakis, and P. K. Shukla, Phys. Plasmas 12, 012310 (2005); P. K. Shukla and M. Khan, ibid. 2, 014504 (2005); T. Tatsuno, M. Ohhashi, V. I. Berezhiani, and S. V. Mikeladze, Phys. Lett. A 363, 225 (2007).

[15] B. Eliasson and P. K. Shukla, Phys. Rev. E 71, 046402 (2005).

[16] A. Luque, H. Schamel, B. Eliasson, and P. K. Shukla, Plasma Phys. Controlled Fusion 48, L57 (2006).
[17] T. Tajima and T. Taniuti, Phys. Rev. A 42, 3587 (1990); P. K. Shukla, N. L. Tsintsadze, and L. N. Tsintsadze, Phys. Fluids B 5, 233 (1993); D. Farina and S. V. Bulanov, Phys. Rev. E 64, 066401 (2001).

[18] H. Schamel and A. Luque, New J. Phys. 7, 69 (2005).

[19] F. Verheest, Phys. Plasmas 13, 082301 (2006).

[20] H. Saleem, Phys. Plasmas 13, 044502 (2006); 14, 014505 (2007).

[21] H. Saleem, J. Vranjes, and S. Poedts, Phys. Lett. A 350, 375 (2006).

[22] R. Sabry, W. M. Moslem, and P. K. Shukla, Phys. Plasmas 16, 032302 (2009)

[23] H. Saleem and N. Batool, Phys. Plasmas 16, 022302 (2009).

[24] V. I. Berezhiani and S. M. Mahajan, Phys. Rev. Lett. 73, 1110 (1994)

[25] V. I. Berezhiani and S. M. Mahajan, Phys. Rev. E 52, 1968 (1995).

[26] S. M. Mahajan and N. L. Shatashvili, Phys. Plasmas 15, 100701 (2008)

[27] N. L. Shatashvili, J. I. Javakhishvili, and H. Kaya, Astrophys. Space Sci. 250, 109 (1997); N. L. Shatashvili and N. Rao, Phys. Plasmas 6, 66 (1999).

[28] A. Esfandyari-Kalejahi, I. Kourakis, M. Mehdipoor, and P. K. Shukla, J. Phys. A 39, 13817 (2006).

[29] V. I. Berezhiani, S. M. Mahajan, Z. Yoshida, and M. Ohhashi, Phys. Rev. E 65, 047402 (2002).

[30] X. L. Chen and R. N. Sudan, Phys. Fluids B 5, 1338 (1993).

[31] N. G. Vakhitov and A. A. Kolokolov, Izv. Vyssh. Uchebn. Zaved., Radiofiz. 16, 1020 (1973); Sov. Radiophys. 9, 262 (1973).

[32] S. M. Mahajan, V. I. Berezhiani, and R. Miklaszewki, Phys. Plasmas 5, 3264 (1998); K. Hayata and M. Koshiba, J. Appl. Phys. 71, 2526 (1991); N. A. Akhmediev and J. M. SotoCrespo, Phys. Rev. A 47, 1358 (1993); V. Skarka, V. I. Berezhiani, and R. Miklaszewski, Phys. Rev. E 56, 1080 (1997). 